\title{
THE INFLAMMATORY RESPONSE OF PULP TISSUE AFTER DIFFERENT DIRECT PULP CAPPING MATERIALS AT DIFFERENT STORAGE TIME
}

\author{
Ali Yakout Dogheim $\mathrm{AY}^{1^{*}}$, Walaa Mohamed Al Samolly² ${ }^{2}$ Khalid Mohamed Noaman ${ }^{3}$, Khadiga Youssef Kawana ${ }^{4}$
}

\begin{abstract}
Objective: Evaluate histologically and compare the inflammatory pulp response following direct pulp capping of mechanically exposed dogs' teeth with: $\mathrm{Ca}(\mathrm{OH}) 2$ base Dycal, UltraBlend Plus and TheraCal LC, at different time intervals. Subjects and methods. A total of 54 permanent teeth of six healthy dogs were used. They were randomly divided into three groups ( $\mathrm{n}=18)$ according to pulp capping material used. Group I: Dycal, Group II: UltraBlend Plus (UBP) and Group III: TheraCal LC. All cavities were restored with Riva LC Resin Modified Glass Ionomer. Each group was subdivided into three subgroups according to storage time $(n=6)$ at time intervals of 1 week, 1 month and 3 months. Control samples ( 3 teeth) were added representing normal untouched teeth for evaluating histological features of normal pulp. Paraffin sections were prepared stained with H\&E then examined under light microscope for histologic evaluation of the inflammatory cell response. Results: Theracal specimens showed the lowest inflammatory score among the three capping materials in one week period followed by UBP and Dycal respectively .The inflammatory score by the end of third month improved for all materials. No inflammation was detected in Theracal specimens by the end of third month. Conclusions: Subsiding of inflammation over the study period is a sign of biological acceptance of the materials used in this investigation. Theracal is superior to Dycal and UBP for pulp capping of mechanically exposed human teeth, it can be used as an effective direct pulp capping material.
\end{abstract}

Keywords: Pulp capping, TheraCal; UltraBlend, Dycal

\section{INTRODUCTION}

Pulp exposure could cause inflammation of the dental pulp (pulpitis), which may become irreversible, causing pain, pulp necrosis, and eventually may require an extraction or root canal treatment. Clinically, the treatment of pulp exposure includes a direct pulp capping to protect the dental pulp and preserve its vitality ${ }^{(1)}$.

The overall aim of direct pulp capping is pulp healing. Several months after direct pulp capping, one of the following effects can be expected either regular pulp tissue without signs of inflammation with a constant layer of reparative dentine, chronically inflamed infiltrated pulp tissue with a permeable layer of reparative dentine interspersed with tunnel defects or highly inflamed pulp tissue with an imperfect, incomplete or missing layer of reparative dentine or a dense collagenous scar tissue in the area of pulp perforation ${ }^{(2)}$.

Multiple pulp capping studies have demonstrated that pulp inflammation can be unrelated to bacterial presence, reinforcing the concept that certain materials applied directly to pulp tissue may elicit

1. Masters Candidate, Department of Operative Dentistry, Faculty of Dental Medicine, Boys, Cairo, Al-Azhar University

2. Lecturer, Department of Operative Dentistry, Faculty of Dental Medicine, Boys, Cairo, Al-Azhar University

3. Professor, Department of Operative Dentistry, Faculty of Dental Medicine, Boys, Cairo, Al-Azhar University

4. Professor, Department of Oral Biology, Faculty of Dental Medicine Alexandria University

-Corresponding author: ali_yakout@hotmail.com 
significant inflammatory response ${ }^{(3)}$. Many studies indicates that calcium hydroxide compounds are the gold standard for pulp capping ${ }^{(4)}$. As a result of its limitations, different formulations of $\mathrm{Ca}(\mathrm{OH}) 2$ have been developed and several dental adhesives have been proposed in direct pulp capping. Hebling $\mathrm{J}$ et al ${ }^{(5)}$ evaluated the cytotoxic effects of resin-based light-cured liners like TheraCal and Ultrablend Plus (UBP), they concluded that Theracal was least cytotoxic when compared to other materials. As the choice between the wide variety of pulp capping materials is somewhat difficult as well as the difference of tissue response after direct pulp capping; the objective of this study was to evaluate histologically and compare the inflammatory pulp response at different time intervals (1 week, 1 month and 3 months) following direct pulp capping of mechanically exposed dogs' teeth with: $\mathrm{Ca}(\mathrm{OH}) 2$ base Dycal, Ultrablend Plus and TheraCal LC.

\section{SUBJECTS AND METHODS}

A total of 54 permanent teeth of six healthy male mongrel dogs aged 18-24 months, and weighing about $18-24 \mathrm{~kg}$ were used in the study. The selected teeth were canine, second, third and fourth premolars and, first molar, they were free from caries, fractures and periodontal disease. The fifty four teeth included in the study were randomly divided into three groups $(\mathrm{n}=18)$ according to pulp capping material used: Group I: Dycal, Group II: UltraBlend Plus and Group III: TheraCal LC. All cavities were restored with Riva LC Resine Modified Glass Ionomer.

Then each group was subdivided into three subgroup according to storage time $(n=6)$ at time intervals of 1 week, 1 month and 3 months. Control samples (3 teeth) were added representing normal untouched teeth for evaluating histological features of normal pulp.

Operations were done under general anesthesia with intramuscular injection of $10 \mathrm{mg} / \mathrm{kg}$ of Ketamine $\mathrm{HCl}$ and $1 \mathrm{mg} / \mathrm{kg}$ of Xylazine. The operation field, dogs' teeth and oral cavity were cleaned and disinfected with $0.2 \%$ Chlorhexidine and rubber dam isolation was performed ${ }^{(6)}$.

A class $\mathrm{V}$ cavity was prepared $1 \mathrm{~mm}$ away from gingival margin on the buccal surface of the teeth until pinkish shadow of the pulp was seen using a bur number 330 in high-speed hand piece with copious water spray. A number one sized round carbide bur was used to drill in the middle of the cavity to expose the pulp in a diameter not exceeding one $\mathrm{mm}$ without impinging the pulp tissue ${ }^{(7)}$.

Pulp capping materials were applied according to manufacturer instructions Dycal was mixed in ratio 1:1 base paste to catalyst paste and placed by burnisher in the cavity not more than $1 \mathrm{~mm}$ in thickness and left 2-6 min, UltraBlend Plus was injected in the cavity at the exposure site and light cured for 20 seconds and Theracal was injected in the cavity at the exposure site and light cured for 20 seconds all materials was placed into the exposed pulp without pushing it into the pulp tissue, materials were randomly assigned into each quadrant.

Post-operative care of dogs included keeping dogs under observation to asses' presence or absence of any infection, they were kept on soft diet and receive a dose of a nonsteroidal anti-inflammatory drug (Rimadyl Carprofen, Zoetis, USA) and have demonstrated acceptable safety profiles to decrease pain postoperatively ${ }^{(8)}$

Two dogs were sacrificed with intravenous anesthetic overdose by injection of concentrated thiopental at the interval 1 week, 1 month and 3 months.

Jaw segments including the operated teeth and parts of surrounding soft tissue were cut out of the animal jaw to be prepared for histological examination. The specimens were fixed in $10 \%$ neural buffered formalin, The specimens were decalcified then in $8 \%$ trichloroacetic acid. The acid was changed every three days for three weeks and specimens were tested for complete decalcification, then processed to paraffin blocks. Serial sections of 
5 microns thickness were prepared and stained with $\mathrm{H} \& \mathrm{E}$ then examined under light microscope for histologic examination. The specimens were examined and evaluated histologically for inflammatory cell response following scoring system used by Nowicka et $\mathrm{al}^{(9)}$.

TABLE (1) Nowicka scoring system.

\begin{tabular}{|l|}
\hline Scores Inflammation intensity \\
\hline 1. Absent or very few inflammatory cells \\
\hline 2. Mild (an average of $<10$ inflammatory cells) \\
\hline 3. Moderate (an average of $10-25$ inflammatory cells) \\
\hline 4. Severe (an average of $>25$ inflammatory cells) \\
\hline Scores Inflammation extensity \\
\hline 1. Absent \\
\hline $\begin{array}{l}\text { 2. Mild (inflammatory cells next to dentin bridge or area of } \\
\text { pulp exposure only) }\end{array}$ \\
\hline $\begin{array}{l}\text { 3. Moderate (inflammatory cells observed in one-third or } \\
\text { more of the coronal pulp or in the midpulp) }\end{array}$ \\
\hline 4. Severe (all of the coronal pulp is infiltrated or necrotic) \\
\hline Dental pulp congestion \\
\hline 1. No congestion \\
\hline $\begin{array}{l}\text { 2. Mild (enlarged blood vessels next to dentine bridge or } \\
\text { pulp exposure) }\end{array}$ \\
\hline 3. Moderate (enlarged blood vessels in coronal pulp) \\
\hline 4. Severe (all coronal pulp is infiltrated with blood cells) \\
\hline
\end{tabular}

\section{RESULTS}

As regards Dycal group: $66.7 \%$ of specimens showed severe inflammatory intensity and extensity together with dental pulp congestion "score 4" in 1 week storage time, while in 3 months storage time inflammatory intensity and extensity and dental pulp congestion were mild "score 2" in $66.7 \%$ of cases. So as a whole the inflammatory response of the pulp improves by time. This was shown by decreasing the inflammatory score from severe at 1 week storage time to moderate at 1 month and finally mild at 3 months storage time.

No statistically significant difference was found between the three studied storage time of Dycal groups as regards inflammatory intensity and extensity but for pulp congestion there was a statistical significant difference between the three studied storage time groups.

For UBP group: Specimens having direct pulp capping with UBP showed a moderate inflammatory intensity $66.7 \%, 83.3 \%$ extensity and dental pulp congestion "score 3 " in $66.7 \%$ in 1 week storage time, while in 1 and 3 months storage time inflammatory intensity was mild "score 2" in 50\% and $66.5 \%$ of cases respectively. The inflammarory extensity and dental pulp congestion were moderate in 1 month storage period in $66.7 \%$ and $83.3 \%$ of specimens respectively, both returned to mild in 3 months storage period in $66.7 \%$ of specimens. No statistical significant difference was present between the different time intervals in UBP group as regards inflammatory intensity, but statistically significant difference between different storage time of the study was detected in UBP as regards inflammatory extensity and congestion.

As regards inflammatory intensity and extensity in theraCal: No significant statistic difference was found between groups. But for dental pulp congestion there was a statistically significant difference between the three time intervals.

Theracal showed the least congestion in 1 week and 1 month period and no congestion in 3 months period compared to Dycal and UBP.

When comparing the three materials: There was a statistically significant difference between theracal and both dycal and UBP as regards inflammatory intensity as well as pulp congestion after three months period

Regarding the effect of capping material on inflammatory score as function of evaluation periods, the data were collected and tabulated in table (2) 
TABLE (2) Prevalence and test of significance for the effect of capping material on inflammatory score as function of evaluation periods.

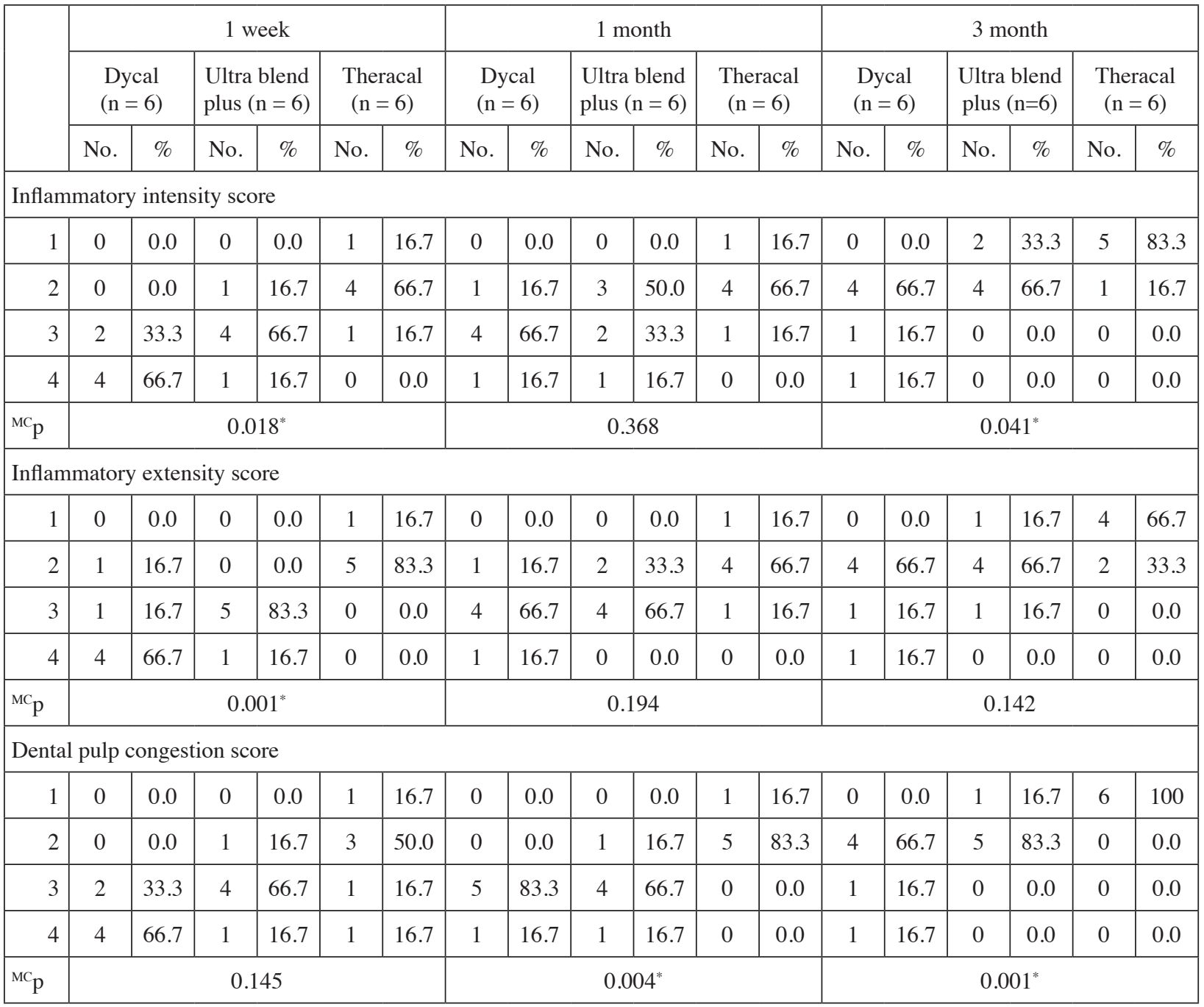

${ }^{\mathrm{MC}} \mathrm{p}$ : p value for Chi square test (Monte Carlo) for comparing between the studied groups

*: Statistically significant at $\mathrm{p} \leq 0.05$

\section{Histological evaluation}

\section{One week (Fig 1,2)}

Dycal group: The histological picture showed loss of the normal architecture of the connective tissue stroma which was heavily infiltrated with inflammatory cells and dilated congested blood vessels. Ruptured blood capillaries with extravasated red blood cells and areas amorphous pale eiosinophilic spaces (edema) were seen.
UBP group: Connective tissue stroma showed multiple vaculations, moderate inflammation with multiple polymorph nuclear leucocytes related to coagulative necrotic areas at the exposure site and dilated blood vessels were seen.

TheraCal group: The histological picture of pulp tissue exhibited alteration in architecture with moderate inflammatory cells and dilated blood vessels otherwise the connective. Tissue stroma showed no changes .. 


\section{One month(fig 3,4)}

Dycal group: Connective tissue stroma showed moderate inflammation was seen with multiple polymorphonuclear leukocytes related to coagulative necrotic area at exposure site.

UBP group: The histological picture of pulp tissue exhibited variable degrees of inflammation reaction, either showing low grade cellular infiltrate with polymorph nuclear leucocytes in connective tissue or even coronal pulp involvement

TheraCal group: Pulp appeared normal in structure with capillaries of different sizes and few scattered in-

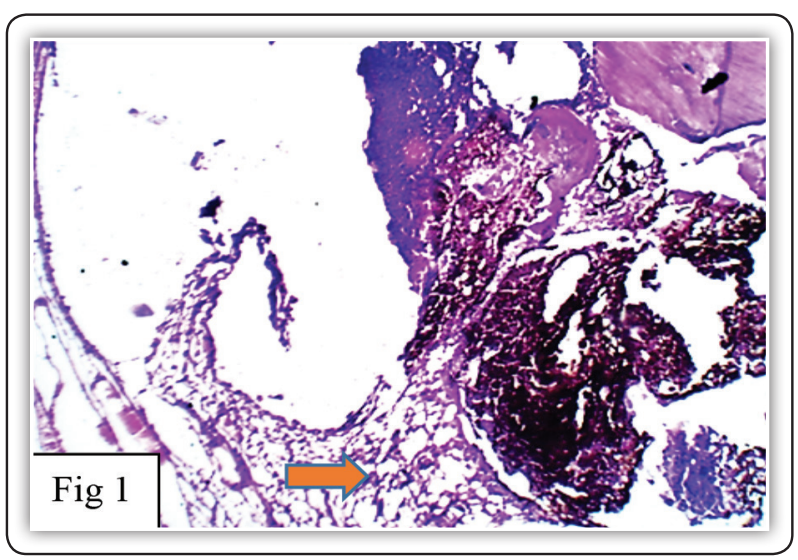

FIG (1) LM. Dycal group one week showing severe inflammatory cell infiltration. (arrow) (H\&E X 100)

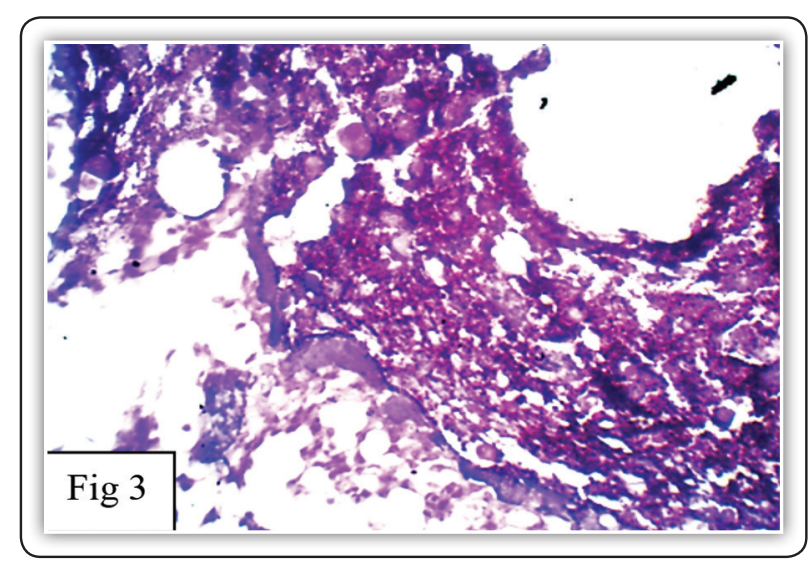

FIG (3) Dycal one month showing moderate inflammation with multiple polymorph nuclear leukocytes. (H\&E X 400). flammatory cells in connective tissue stroma..

\section{Three months (fig 5,6)}

Dycal group: dilated congested blood vessels and moderate inflammatory cellular infiltrate were seen in connective tissue stroma.

UBP group: Mild inflammatory cellular infiltration was seen, polymorph nuclear leucocytes were traced in the deep layers of the pulp connective tissue stroma..

TheraCal group: normal connective tissue stroma with mild inflammatory cell infiltrate.

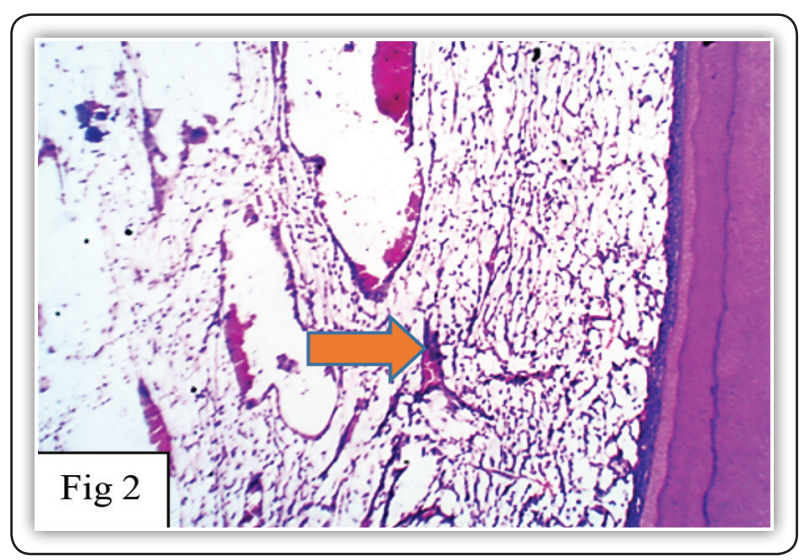

FIG (2) L.M. UBP group one week showing; moderate inflammatory cells infiltration multiple polymorph nuclear leucocytes. (arrow) (H\&E X 100)

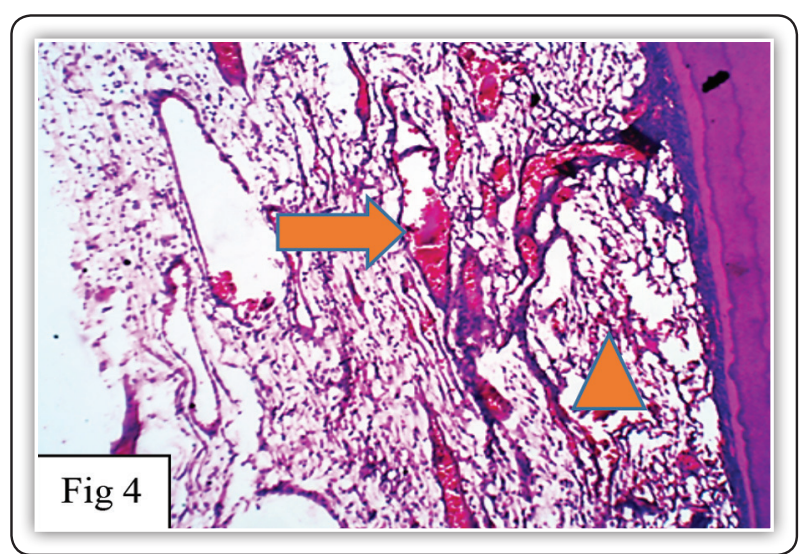

FIG (4) L.M. Ultra Blend Plus one month showing moderate cell infiltration with polymorph nuclear leucocytes(arrow head). among disorganized pulp structure with dilated blood vessels. (arrow). (H\&E X 100) 


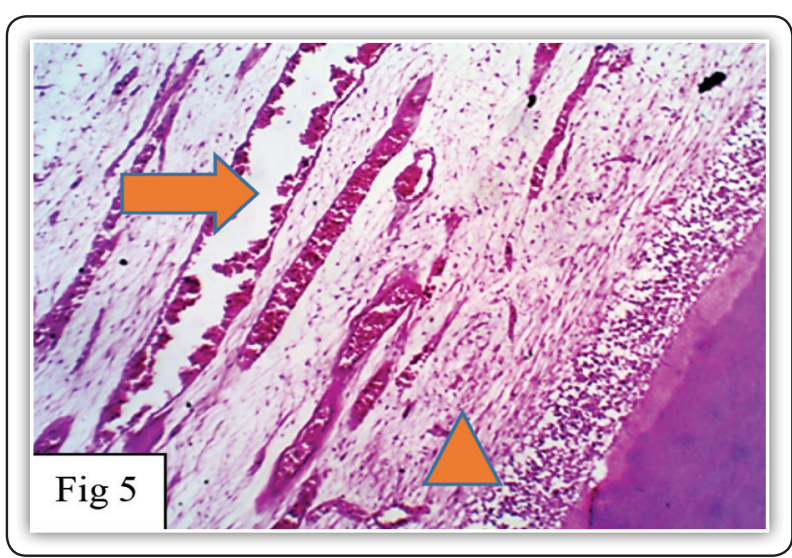

FIG (5) L.M. Dycal three months showing; dilated congested blood vessels(arrow). with moderate inflammatory cell infiltrate. (arrow head). (H\&EX100)

\section{DISCUSSION}

In the last decade, many experimental and clinical studies have been carried out to develop and test new dental materials endowed with safe biocompatibility and anti- infective properties ${ }^{(7)}$. Direct pulp capping (DPC) has been frequently used to preserve vitality of dental pulp ${ }^{(8)}$. The material used should provide an appropriate host response. This means that the tissues that come in contact with the materials should not show any toxic, irritating, inflammatory, allergic, genotoxic or carcinogenic response ${ }^{(5)}$.

Nowika et $\mathrm{al}^{(12)}$ and Suzuki et $\mathrm{al}^{(13)}$ stated that in vivo studies are required to understand pulp response when dental materials are used for direct pulp capping .

Negma et al and Matsuo et al examined the length of time necessary for adequate postoperative follow up, and suggested that 3 months period was adequate for a tentative prognosis this was also choosen for our study. ${ }^{(14,15)}$

Dog was the selected animal model as the mechanisms of induction and synthesis of dentin in this animal are the same as in human beings ${ }^{(16)}$. The bleeding was controlled by rinsing the exposure site with sterile saline solution this method was also adapted by Al-sherbiny et al ${ }^{(17)}$.

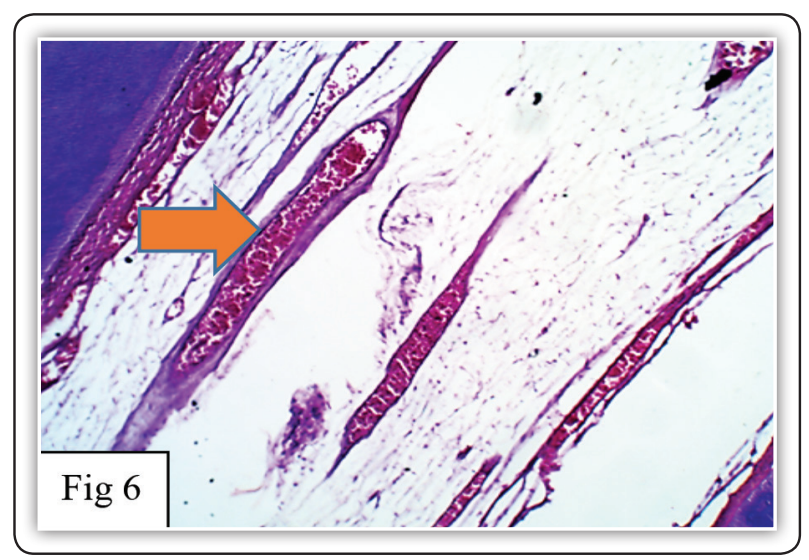

FIG (6) L.M TheraCal LC three months showing normal connective tissue stroma with dilated congested blood vessels (arrow).Opposite the exposure site. (H\&Ex100)

Godoy and Murray concluded that bleeding control are most likely even more important to preserve pulp vitality than is the choice of a specific direct pulp capping agent, because of evidence that pulp healing is most compromised by hemorrhagic injury $^{(18)}$.

In order to assess the biological capability of the tested materials, histopathological evaluation was performed to determine the inflammatory reaction of pulp tissues over storage time of 1 week, 1 month, and 3 months to evaluate the pulp response to the three used capping materials: Dycal, Ultrablend plus and Theracal. The grading of their success was based on: pulp vitality and evaluation of pulp inflammatory score: the inflammatory intensity and extensity scores as well as pulp congestion

In one week, the inflammatory intensity and extensity scores had statistically significant difference between all three materials used showing severe inflammatory intensity and extensity of dycal compared to ultrablend plus and theracal . Aeinehchi et al in $2003^{(19)}$ studied the effect on pulp that was capped with calcium hydroxide cement and they were extracted after periods of 1 week, 2 months, 3 months, 4 months and 6 months. Histological evaluation demonstrated that the inflammatory response lasted for 6 months, starting 
with acute inflammation and ending in mild chronic inflammation. This was also proved by Stefanova et al ${ }^{(20)}$ and was in concordance with our study.

Chen and Suh ${ }^{(21)}$ on the other hand, suggested that light curable resin modified calcium silicates, such as Theracal LC, have less cytotoxicity and better or comparable clinical outcomes during the 1st week of DPC.

Current study showed that in one month, a reduction in the inflammatory response was evident in all groups. This represented a positive healing outcome. The results were statistically significant between the three materials after one month as regards inflammatory extensity and pulp congestion.

These findings are in agreement with Maden et al (22) and Modena, K.C ${ }^{(23)}$ who described the inflammatory changes encountered with dycal and UBP and attributed them to be responsible for the pulp repair and hard tissue barrier formation. They attributed this to the high $\mathrm{pH}$ which induces a coagulation necrosis layer when in direct contact with pulp tissue.

The results of the present study revealed that TheraCal LC caused inflammation with varying intensities which was a normal response to a foreign material and subsided at 3 months interval. This may be attributed to excellent sealing properties which prevent microleakage and pulpal inflammation by providing a predictable secondary barrier ${ }^{(24)}$.

In accordance with the previous studies, samples that were capped with Dycal showed less satisfactory results ${ }^{(25,26)}$.

The present histological analysis of direct capping with the three different materials confirms that the inflammation of the pulp is reversible. The few areas of inflammatory infiltrate observed and the small layer of pulp necrosis at one week, are probably caused by the preparation of the cavities or the penetration of the bur in the pulp, and it is not caused by different materials
Most of the published studies Alquahtani et al (27) Goldberg et al ${ }^{(28)}$ reported that the healing sequence starts with an initial moderate inflammatory process, and now there are evidences that inflammation is a prerequisite for tissue healing as a first step, followed by pulp regeneration, also described as pulp repair.

The presence of inflammation of the pulp until the longest storage time 3 months after capping although diminished yet it was a common feature in Dycal specimens; as stated by Parolia et $\mathrm{al}^{(4)}$ and Nair et $\mathrm{al}^{(29)}$.

Goldberg et al ${ }^{(28)}$ stated that light curable resin based cements have been introduced to enable a better marginal seal and lesser dissolution; this may explain the reduction in inflammation at 3 months for UBP.

As regards Theracal, Lee et al ${ }^{(25)}$ considered the absence of polymorphonuclear leucocytes, a sign of acute inflammation, in favor of TheraCal LC which also correlates to clinical findings where no abscess formation was evident. TheraCal LC histologically appeared to be more pulpally kind as compared to other groups with very mild pulpal inflammation and minimal necrosis but there were no statistically significant differences between the groups in regard to pulpal inflammation $(\mathrm{P}=1.00)^{(11)}$.

Pulp tissue in the present study displayed intense to moderate inflammation with all the test materials at 1 weeks, then significant improvement occurred by 3 months, coinciding with the findings of Sadek $\mathrm{H}$ and Abou el Nasr H. ${ }^{(30)}$.

\section{CONCLUSIONS}

1. The subjacent pulp tissue play a role in pulp healing together with the pulp capping agents which help the process.

2. Calcium hydroxide based chemical cure cement (Dycal) evoked severe inflammatory response in the pulp. However it subsides over the study period which is a sign of biological acceptance. 
3. Histological evaluation of pulp treated teeth using TheraCal LC in animals have shown interesting and promising results that encouraged its use in human teeth.

4. Theracal and UBP share the indications and mode of action with Dycal but does not have its drawbacks, they can be used as an effective direct pulp capping material.

\section{RECOMMENDATIONS}

1. Although the short term results of TheraCal LC are promesing, the long term efficacy remains limited so, we need to study longer periods.

2. Studying the effect of direct capping on larger scales with larger sample size.

\section{REFERENCES}

1. Hanada K, Morotomi T, Washio A, Yada N, Matsuo K, Teshima $\mathrm{H}$, et al. In vitro and in vivo effects of a novel bioactive glass-based cement used as a direct pulp capping agent. Journal of Biomedical Materials Research Part B: Applied Biomaterials 2019;107:161-8

2. Dammaschke T, Leidinger J, Schäfer E. Long-term evaluation of direct pulp capping - treatment outcomes over an average period of 6.1 years: Clinical Oral Investigations; 2009

3. T. J. Hilton. Keys to Clinical Success with Pulp Capping: A Review of the Literature: Restorative Dentistry: 2009 Sep;34 (5):615-625.

4. Parolia A, Kundabala M, Rao N, Acharya S, Agrawal P, Mohan M, et al. A comparative histological analysis of human pulp following direct pulp capping with Propolis, mineral trioxide aggregate and Dycal. Australian Dental Journal. 2010;55(1):59-64.

5. Hebling J, Lessa FCR, Nogueira I, Carvalho RM, Costa CAS. Cytotoxicity of resin-based light-cured liners. American Journal of Dentistry 2009;22:137-42.

6. Asgary S, Kamrani FA. Antibacterial effects of five different root canal sealing materials. Journal of oral science. 2008;50(4):469-74.

7. Tziafa C, Koliniotou-Koumpia E, Papadimitriou S, Tziafas D. Dentinogenic activity of biodentine in deep cavities of miniature swine teeth. Journal of endodontics 2015;41:1161-6.
8. Hellyer, Peter, et al. "AAHA/AAFP pain management guidelines for dogs and cats. Journal of Feline Medicine \& Surgery 9.6 (2007): 466-480.

9. Nowicka A, Lipski M, Parafiniuk M, Sporniak-Tutak K, Lichota D, Kosierkiewicz A, et al. Response of human dental pulp capped with biodentine and mineral trioxide aggregate. J Endod 2013;39:743-7.

10. Poggio C, Arciola CR, Beltrami R, Monaco A, Dagna A, Lombardini $\mathrm{M}$, et al. Cytocompatibility and antibacterial properties of capping materials. ScientificWorldJournal 2014;2014:181945.

11. Aranha AMF, Giro EMA, Hebling J, Lessa FCR, Costa CAdS. Effects of light-curing time on the cytotoxicity of a restorative composite resin on odontoblast-like cells. Journal of Applied Oral Science 2010;18:461-6.

12. Nowicka A, Łagocka R, Lipski M, Parafiniuk M, Grocholewicz K, Sobolewska E, et al. Clinical and histological evaluation of direct pulp capping on human pulp tissue using a dentin adhesive system. BioMed research international 2016; (4):1-9.

13. Suzuki M, Kato C, Kawashima S, Shinkai K. Clinical and histological study on direct pulp capping with $\mathrm{CO} 2$ laser irradiation in human teeth. Operative Dentistry. 2019;44(4):336-47.

14. Negm AM, Hassanien EE, Abu-Seida AM, Nagy MM. Biological evaluation of a new pulp capping material developed from Portland cement. Experimental and Toxicologic Pathology. 2017;69(3):115-22.

15. Matsuo T, Nakanishi T, Shimizu H, Ebisu S. A clinical study of direct pulp capping applied to carious-exposed pulps. Journal of endodontics. 1996;22(10):551-6.

16. El Ashry SH, Abu-Seida AM,Bayoumi AA, Hashema AA. Regenerative potential of immature permanent non-vital teeth following different dentin surface treatments. Experimental and Toxicologic Pathology 2016;(68):181-190

17. Al-Sherbiny IM, Abu-Seida A, Farid M, Motawea I, Bastawy H. Histopathological pulp response of dog's teeth capped with biosealer and biodentine: An in vivo study . Saudi Endodontic Journal 2020. 10(3):226-233

18. Godoy F, Murray P. Systemic evaluation of various haemostatic agents following local application prior to direct pulp capping. Braz J Oral Sci. 2005 4(14):791-797

19. Aeinehchi M, Eslami B, Ghanbariha M, Saffar AS. Mineral tri- oxide aggregate (MTA) and calcium hydroxide as pulp capping agents in human teeth: a preliminary report. Int Endod J. 2003;36:225-31. 
20. Stefanova V, Tsanova S, Atanasova P, Borisov I, Chaprazov T, Tsanova M. early resultsafter direct pulp capping withticalcium silicate based material and and mieral trioxide aggregate and ER: YAG cavity preparation in dogs. Trakia Journal of Sciences. 2014;12(1):331-4.

21. Chen L, Suh BI. Cytotoxicity and biocompatibility of resin-free and resin-modified direct pulp capping materials: A state-of-the-art review. Dent Mater J. 2017;36:1-725.

22. Maden M, Orhan EO, Ertgul E F, Sengüven B. The inflammatory response of the pulp after direct capping with platelet-rich plasma and enamel matrix derivative: A controlled animal study. Open Journal of Stomatology. 2014;(4):01:8.

23. Modena KCdS, Casas-Apayco LC, Atta MT, Costa CAdS, Hebling J, Sipert CR, et al. Cytotoxicity and biocompatibility of direct and indirect pulp capping materials. Journal of Applied Oral Science. 2009;17:544-54.

24. Cannon M, Gerodias N, Vieira A, Percinoto C, Jurado R. Primate pulpal healing after exposure and TheraCal application. Journal of Clinical Pediatric Dentistry. 2014;38(4):333-7.

25. Lee H, Shin Y, Kim S-O, Lee H-S, Choi H-J, Song JS. Comparative Study of Pulpal Responses to Pulpotomy with ProRoot MTA, RetroMTA, and TheraCal in Dogs' Teeth. Journal of Endodontics. 2015;41(8):1317-24.

26. Wassel MO, Amin DH, Badran AS. Clinicall, radiographic, and histological evaluation of theracal pulpotomy in human primary teeth. Egyptian Dental Journal.2017;63:365-75

27. Alqahtani AR, Yaman P, McDonald N, Dennison J. Efficacy of calcium hydroxide and resin-modified calcium silicate as pulp-capping materials: a retrospective study. General dentistry. 2020;68(6):50-4.

28. Goldberg M, Njeh A, Uzunoglu E. Is Pulp Inflammation a Prerequisite for Pulp Healing and Regeneration? Mediators of Inflammation. 2015;2015:347649.

29. Nair P, Duncan H, Pitt Ford T, Luder H. Histological, ultrastructural and quantitative investigations on the response of healthy human pulps to experimental capping with mineral trioxide aggregate: a randomized controlled trial. International endodontic journal. 2008;41(2):128-50.

30. Sadek H, Abou El Nasr H. Histologic assessment of pulp response to strontium ranelate and bisphospho-nates when compared to calcium hydroxide as direct pulp capping materials. An animal study. Egyptian Dental Journal. 2020;66:1403-12. 